\title{
Deciphering Location Context - A Semantic Web Approach
}

\author{
Zhenning Shangguan ${ }^{1,2}$ and Deborah L. McGuinness ${ }^{2}$ \\ ${ }^{1}$ Pitney Bowes, 27 Waterview Drive, Shelton, CT 06484, USA \\ zhenning.shangguan@pb.com \\ ${ }^{2}$ Rensselaer Polytechnic Institute, $1058^{\text {th }}$ Street, \#2104, Troy, NY 12180, USA \\ dlmecs.rpi.edu
}

\section{Introduction}

Mobile user location data has been commercially exploited and studied due to the commoditization of GPS position sensors and the popularity of Location Based Services (LBS). Context researchers have already studied how to understand human mobility using location histories [1], and how to model location context using ontologies [2]. However, these studies make surprisingly little use of rich geospatial data and knowledge about the world to a) explicitly describe user locations, and b) possibly infer implicit contexts. In this paper, we demonstrate that openly accessible geospatial data can facilitate both a) and b), thus resulting in improved understanding of mobile user location context.

\section{Approach}

We aim to understand the user's location context leveraging rich semantic geospatial data about the world. More concretely, we seek 1) explicit information that describes the user's current location point and surroundings, and 2) implicit insights that describe the user's activity status, situation, or intent. We call the former static location context, and the latter dynamic location-informed user context. Being critical information that can be used to characterize the situation of the user, both are considered to be the constituent parts of what we refer to as location context. An example scenario includes a particular mobile user's current location with timestamp, e.g., $<$ latitude $=41.055861$, longitude $=-73.517434$, timestamp $=2013-02-15$ 08:30:00 EST-0500>, and optionally some prior knowledge about the user, such as his home city of Stamford, CT, and work city of Shelton, CT. In this case, we know that his current position is on Highway Interstate 95 (I-95) North Bound (static location context), and we can also infer that he is on his way to work (dynamic location-informed user context). We build a geospatial knowledge graph to aid in resolving static location context. Dynamic location-informed context is obtained using SPARQL queries extended with geospatial functionalities.

Static Location Context. Fig. 1 shows the graph representation of a small part of Linked Geo Data (LGD) [3] used in context understanding. We also integrate proprietary commercial geospatial data with LGD, resulting in a much larger knowledge base 


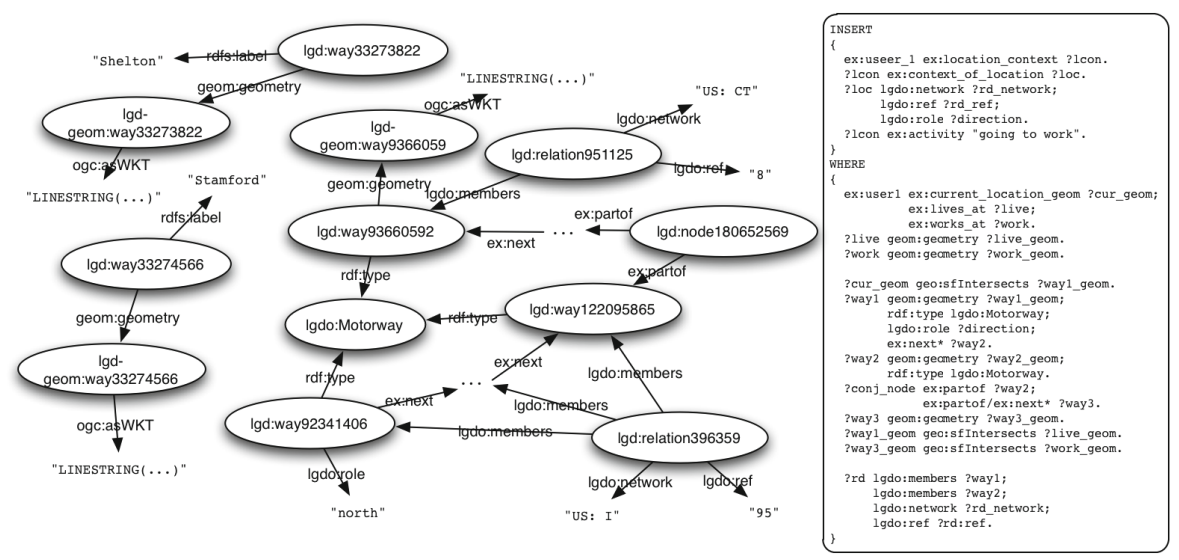

Fig. 1. Example Geospatial Knowledge Graph and SPARQL Query

(currently over 700 million triples) with socioeconomic data and geographic boundaries of neighborhoods, businesses, and residential properties.

Explicit Location-Derived User Contexts. The geospatial knowledge graph enables various common sense heuristics for understanding user contexts to be formally expressed in the form of SPARQL queries. Fig. 1 shows a sample SPARQL query to understand the user's contexts discussed in the previous example. Notice how geospatial operations and property paths are used to retrieve his static location context (i.e., Highway Interstate 95 (I-95) North Bound) and to infer dynamic context (i.e., "going to work") given the former. Please note that, for illustration purposes, geospatial operations are expressed using GeoSPARQL, which is currently not standardized.

\section{Conclusion and Future Work}

We proposed and demonstrated the feasibility of a novel approach to understand mobile user location context leveraging SPARQL and geospatial data available in the web of Linked Open Data. Future work includes a semantic model of a person's location context, and spatial-temporal context inference mechanisms.

\section{References}

1. González, M.C., Hidalgo, C.A., Barabási, A.-L.: Hidalgo, and Albert-László Barabási: Understanding Individual Human Mobility Patterns. Nature 453, 779-782 (2008)

2. Chen, H., Finin, T., Joshi, A.: An Ontology for Context-Aware Pervasive Computing Environments. Special Issue on Ontologies for Distributed Systems, Knowledge Engineering Review 18, 197-207 (2004)

3. Stadler, C., Lehmann, J., Höffner, K., Auer, S.: LinkedGeoData: A Core for a Web of Spatial Open Data. Semantic Web Journal (2012) 